\title{
Review Article \\ Derailing the UPS of Protein Turnover in Cancer and other Human Diseases
}

\author{
Jit Kong Cheong ${ }^{1}$ and Stephen I-Hong Hsu' \\ ${ }^{1}$ Cancer and Stem Cell Biology Program, Duke-NUS Graduate Medical School, 8 College Road, Singapore 169857 \\ ${ }^{2}$ Sid Martin Biotechnology Incubator, University of Florida, 12085 Research Drive, Alachua, FL 32615, USA
}

Correspondence should be addressed to Jit Kong Cheong; jitkong.cheong@duke-nus.edu.sg

Received 27 March 2013; Revised 1 July 2013; Accepted 15 July 2013

Academic Editor: Jianming Ying

Copyright (C) 2013 J. K. Cheong and S. I.-H. Hsu. This is an open access article distributed under the Creative Commons Attribution License, which permits unrestricted use, distribution, and reproduction in any medium, provided the original work is properly cited.

Protein modifications by the covalent linkage of ubiquitin have significant involvement in many cellular processes, including stress response, oncogenesis, viral infection, transcription, protein turnover, organelle biogenesis, DNA repair, cellular differentiation, and cell cycle control. We provide a brief overview of the fundamentals of the regulation of protein turnover by the ubiquitin-proteasome pathway and discuss new therapeutic strategies that aim to mitigate the deleterious effects of its dysregulation in cancer and other human disease pathophysiology.

\section{Introduction}

The timely degradation of many transiently induced and/or oscillatory proteins is fundamental to the maintenance of diverse biological processes, including receptor trafficking, cell cycle progression, DNA repair, gene transcription, autophagy, and programmed cell death [1-7]. Dysregulation of such regulatory mechanisms has been reported to underlie a growing list of human diseases including cancers, neurodegenerative diseases, and inflammatory and autoimmune disorders. Clearance of intracellular proteins in the cell occurs primarily in three highly specialized subcellular organellesthe proteasome, the lysosome, and the autophagosome. A small protein modifier, ubiquitin, appears to be the common denominator in the targeting of substrates to these degradation pathways in mammalian cells. In this review, we discuss the molecular basis of the ubiquitin-proteasome degradation pathway, the human diseases commonly associated with its dysregulation, and the potential for pharmacologic targeting of the ubiquitin-proteasome pathway as a novel therapeutic approach.

\section{Biological Functions of the Ubiquitin-Proteasome System (UPS)}

2.1. The Core UPS Machinery. As depicted in Figure 1, ubiquitination of target proteins is achieved through the functional orchestration of three classes of enzymes: E1 (ubiquitinactivating enzyme), E2 (ubiquitin-conjugation enzyme), and E3 (ubiquitin ligase). Ubiquitin, in an initial energydependent step, associates with these enzymatic components through a labile thioester linkage. This facilitates its covalent ligation to the target through a more stable isopeptide linkage to the $\mathcal{\varepsilon}$-amino group of acceptor lysine residues or, less frequently, the amino terminus. The enzymatic cascade of ubiquitination is characterized by extensive combinatorial complexity and specificity, as dictated by the diversity of its constituent enzymes: two known E1s, tens of E2s, and hundreds of E3s [2].

Ubiquitin ligases may exist as multiprotein complexes or as single proteins, and they may contain one of a number of domains that promote ubiquitination, such as the RING domain or HECT domain [8]. The Cullin RING ligases (CRLs) represent the largest subclass of E3 enzymes and function as multiprotein complexes. In their simplest form, they are decorated with a RING domain-containing protein (RBX1 or RBX2), a regulatory Cullin, and a substrate-binding adaptor. In addition, a growing list of linker/adaptor proteins has been found to be associated with the core components of ubiquitin ligase to increase their substrate coverage. For instance, there are 69 mammalian F-box proteins that act as substrate-binding adaptors to the Skp-Cullin-F-box (SCF) E3 ligase complex [2]. 


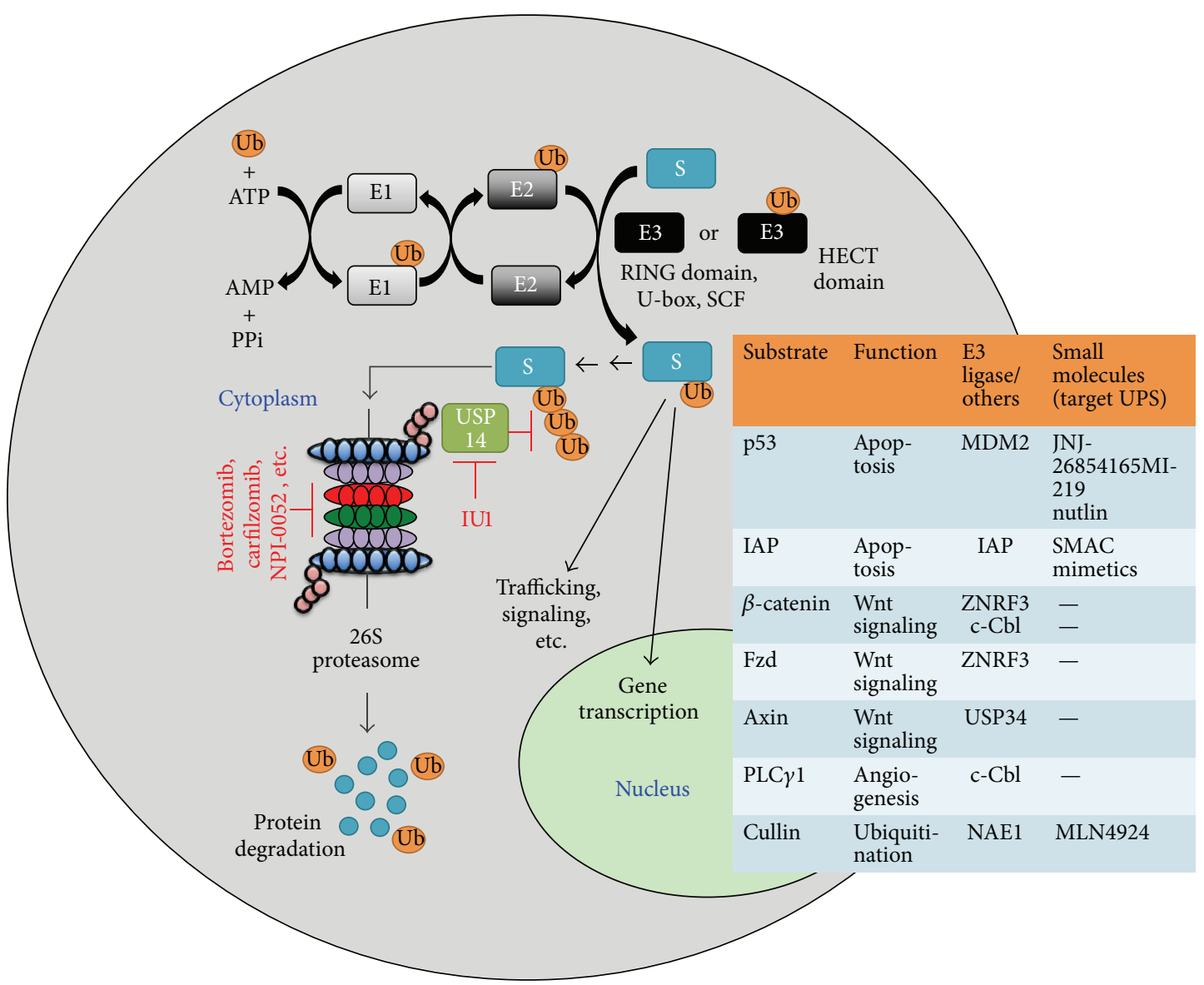

FIGURE 1: Targeting the UPS of protein turnover in cancer and other human diseases. Ubiquitination of target proteins is achieved through the functional orchestration of three classes of enzymes: E1 (ubiquitin-activating enzyme), E2 (ubiquitin-conjugation enzyme), and E3 (ubiquitin ligase). Examples of known and new UPS substrates discussed in this review and their targeting small molecules are listed in the accompanying table. S: substrate; Ub: ubiquitin.

The other key component of the UPS is the barrel-shaped $26 \mathrm{~S}$ proteasome. It is composed of a core (20S) subunit containing multiple proteolytic sites and a 20 S regulatory subunit that controls the access to the core subunit. The $20 \mathrm{~S}$ core subunit is made up of an axial stack of four heptameric rings: two opposing rings stacked between two outer rings. Each $\alpha$ - or $\beta$-ring contains seven unique subunits ( $\alpha_{1}$ through $\alpha_{7}$ and $\beta_{1}$ through $\beta_{7}$ ). The $20 \mathrm{~S}$ proteasome, whose components are less defined, recognizes and removes the ubiquitin chains on target substrates, unfolds the resulting deubiquitinated proteins, and channels the unfolded polypeptides into the proteolytic chamber of the $20 \mathrm{~S}$ core subunit. Proteasome proteolytic sites are located in the interior face of the $\beta$ rings of the 20S. The $\beta_{5^{-}}, \beta_{2^{-}}$, and $\beta_{1}$-subunits are responsible for the chymotrypsin-like, trypsin-like, and caspaselike (also known as peptidylglutamyl-peptide hydrolase) activities, respectively. The $\alpha$-rings serve as the gate to control the entry of polypeptides into the proteolytic chamber and regulate the exit of proteolytic products. To gain entry into the proteolytic sites of the proteasome, protein substrates must be unfolded by a hexamer of ATPases associated with the base of the regulatory subunit. Other components of the regulatory subunit have also been implicated in the recruitment of a wide variety of protein substrates [9]. Notably, three deubiquitinating enzymes (DUBs), POH1/PSMD14, USP14, and UCH37 (Ubp6 and Rpnll in budding yeast), are found to be associated with the mammalian regulatory subunit of the proteasome. Like their ligase counterparts, these DUBs have distinct specificities for different ubiquitin chain linkages to recycle ubiquitin in the cell. The DUBs fall into five major classes: four cysteine (Cys) protease classes and one metalloprotease class [10]. The role of reversible ubiquitination in a diverse range of important biological processes has attracted intense interest in the identification and characterization of human diseases in which dysregulation of DUB-mediated pathways is an underlying defect that may be amenable to therapeutic targeting.

2.2. Diverse Repertoire of Ubiquitin Signals in the Cell. Ubiquitin, a small 76-residue protein, possesses seven critical lysine residues (K6, K11, K27, K29, K33, K48, and K63) and an amino-terminal (N-terminal) methionine residue (M1) that conjugates with the carboxyl-terminal (C-terminal) glycine of ubiquitin moieties in chain assembly and extension to generate polymers. Although ubiquitin has a relatively rigid globular structure, it remains one of the most versatile signaling 


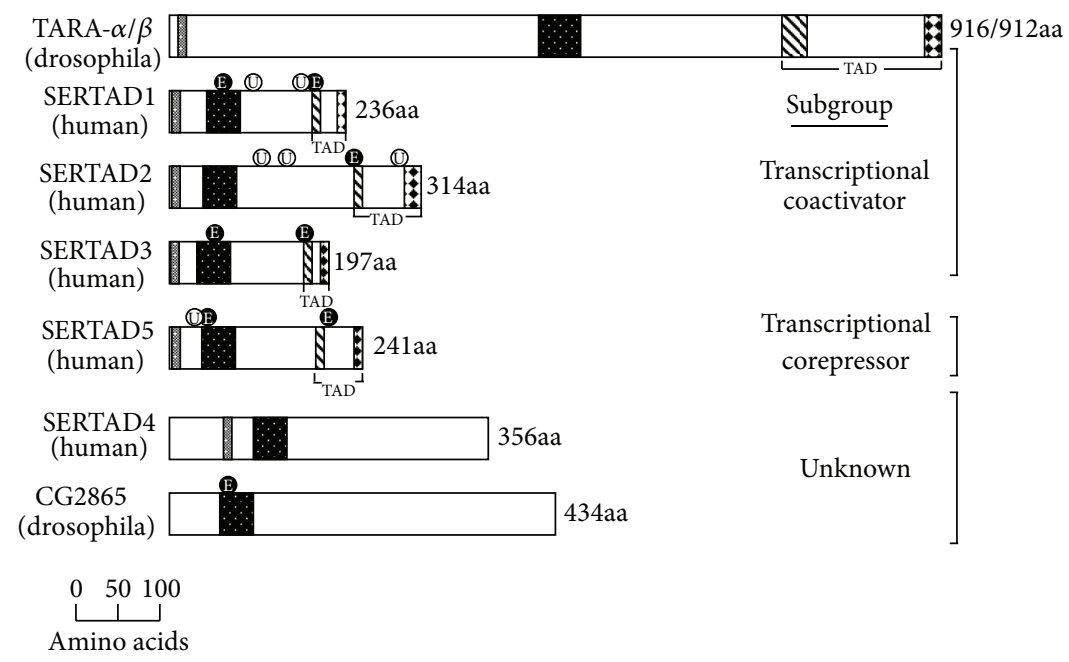

Cyclin A-binding domain/NLS
SERTA domain
$\mathbb{N}$ PHD-bromo interaction domain

C-terminal homology domain

(B) NES

(1) CUE domain

FIGURE 2: Domain organization and conservation of the SERTAD protein family. Sequences were analyzed and aligned using BLAST/ ClustalW algorithms. The GenBank (NCBI) accession numbers used are as follows: human SERTAD1 NP_037508, human SERTAD2 NP _ 055570, human SERTAD3 AAH50643, human SERTAD4 CAB81635, human SERTAD5 NP_060425, Drosophila TARA $\alpha / \beta$ AAF43019/ AAF43018, and Drosophila CG2865 AAL13703. Putative ubiquitination sites (CUE domains) are identified in SERTAD1 (aa94-104 and aa160166), SERTAD2 (aa132-136, aa159-163, and aa273-280), and SERTAD5 (aa24-34).

molecules in the cell. Despite polar residues dominating the surface of ubiquitin, its transient and noncovalent interaction with most ubiquitin-binding domains (UBDs) appears to be solely mediated by a few hydrophobic patches [7, 11]. Ubiquitin can bind to UBDs as monomers (in a process known as monoubiquitination) or they can be packed into polymeric chains that are flexible (as in the case of K63- or M1-linked chains) or rigid (as in the case of K48- or K11-linked chains) $[8,12-15]$. To bring diversity to the repertoire of ubiquitin signaling in the cell, these polyubiquitin chains can be conjugated via the same linkages (homotypic) or mixed linkages (heterotypic) and are frequently varied in their lengths. These polyubiquitin chains serve as a recognition motif that allows a subset of protein substrates to be escorted to the $26 \mathrm{~S}$ proteasome via a unique set of proteins, including the chaperone CDC48/p97 [16].

Ubiquitin-mediated proteolysis functions to regulate the rapid turnover of many transcription factors and co-regulators. A classic example is the regulation of the expression of cyclin E during normal cell cycle progression. $\mathrm{SCF}^{\mathrm{FBW} 7}$ is the specific E3 ubiquitin ligase that binds cyclin E and escorts it to the 26S proteasome. Fbw7 is the substrate recognition subunit of $\mathrm{SCF}^{\mathrm{FBW} 7}$. During the identification and characterization of the transcriptional co-regulatory/integrator proteins SERTAD1 and SERTAD2 (Figure 2) as components of an active transcription complex with E2F1/DP1, we observed that antagonism of the integrator function of the SERTAD proteins led to aberrant cyclin E accumulation, leading to Geminin deregulation and cell death due to the loss of genomic stability $[17,18]$. Our studies identified
Fbxw7, encoding Fbw7, as a novel E2F-responsive gene that is co-regulated by the SERTAD proteins. Protein decay analyses indicated that the accumulation of Geminin was due to protein stabilization. The latter implicates decreased activity of anaphase promoting complex/cyclosome (APC/C), the E3 ubiquitin ligase that mediates its degradation. Thus, a complex interplay mediated by multiple E3 ubiquitin ligases serves to couple the SERTAD1 and SERTAD2 proteins in their function as transcriptional integrators and co-regulators on E2F-responsive gene promoters, with regulation of cyclical expression and degradation of cyclin $\mathrm{E}$ and Geminin levels by $\mathrm{SCF}^{\mathrm{FBW}}$ and APC/C E3 ligases, respectively, to ensure proper replication of DNA and maintenance of genomic stability.

The SERTAD1 and SERTAD2 proteins are themselves subject to complex regulation in function [19] and degradation [20], as the domain that signals their ubiquitinationthe "degron"-overlaps closely with a transcriptional activation domain (TAD), revealing a close correlation between regulation of transcriptional activity and proteolysis [21]. The VP16 TAD has been shown to signal ubiquitination through the Met30 substrate recognition component of the SCF ubiquitin-ligase family, which is required for the VP16 TAD to activate transcription [22], leading to a proposal that ubiquitination regulates TAD function by serving as a dual signal for activation and activator destruction. One group has shown that the SCF (Met30) ubiquitin ligase, which targets the transcription factor Met4 for inactivation as a mechanism for maintaining normal cell cycle progression in budding yeast [23], does so through the attachment of a single ubiquitin chain at K163 in Met4 [24]. This ubiquitin linkage alters 
Met 4 activity but does not play a role in proteasome-mediated degradation. The independent attachment of a ubiquitin chain structure at K48 is required for Met4 substrate targeting to the $26 \mathrm{~S}$ proteasome. The same group identified a UBD in Met4 that restricts the length of the polyubiquitin chain assembled on Met4 and prevents proteasomal recognition and degradation of polyubiquitinated Met4 [25]. In addition, a growing body of literature supports the ability of ubiquitin to bind noncovalently with $\mathrm{UBD}$ and $\mathrm{SH} 3$ domain-containing proteins [26-28].

The CUE domain is one of the first ubiquitin-interacting/associating domains to be described and is characterized by an invariant proline followed by a highly conserved di-leucine motif [29]. Notably, putative CUE domains exist in several SERTAD protein family members [20, 30]. As shown in Figure 2, CUE domains are identified in SERTAD1 (aa94-104 and aa160-166), SERTAD2 (aa132-136, aa159-163, and aa273-280), and SERTAD5 (aa24-34). Ongoing studies will thus shed light on the role of these CUE domains in the regulation of the SERTAD family of transcriptional coregulators and their influence on the outcome of human diseases like cancer, obesity, and type 2 diabetes [31-33].

2.3. Ubiquitin-Binding Domains (UBDs). Ubiquitin signaling transduction relies on the noncovalent interaction of UBDs with mono- or polyubiquitin chains. To date, more than 20 UBDs have been identified among protein sequences in the mammalian proteome. They are known to possess important structural folds for protein-protein interactions, including $\alpha$ helical structures, zinc fingers, and pleckstrin homology $(\mathrm{PH})$ folds [11]. In general, isolated UBDs preferentially interact with monoubiquitin via a conserved hydrophobic patch surrounding isoleucine 44 . The measured affinity of isolated UBDs for monoubiquitin frequently falls in the micromolar range in vitro [7, 11]. However, such affinity of UBDcontaining proteins for monoubiquitin in the cell may likely differ as a result of influence by a variety of factors, such as the presence of tandem copies of one UBD in the same protein, its oligomerization, and its subcellular localization. In addition, many UBDs have been shown to exhibit selectivity for certain types of polyubiquitin chains $[7,11,13]$. The linkage selectivity may also arise from multivalent binding between tandem UBD arrays in a given protein and ubiquitin monomers or linkages in a polyubiquitin chain $[34,35]$. The function of tandem UBD arrays has been recently hypothesized to increase the affinity for a given ubiquitinated substrate rather than simultaneously engaging multiple substrates.

2.4. Specificity of Ubiquitin Signaling. Distinct ubiquitin signals have long thought to play unique roles in specific cellular functions. For instance, K48-linked polyubiquitin chains (also known as the "classical" ubiquitin chains) were originally described as the signal that earmarks substrates for proteasomal degradation. The nonclassical K11-, K63-, or M1linked ubiquitin chains, on the other hand, have been shown to be involved in many other biological processes such as cell cycle regulation, DNA repair, and inflammatory responses [6,13-15]. However, several reports challenged this paradigm by demonstrating that these ubiquitin signals may play nonmutually exclusive roles. For example, the APC/C generates the nonclassical K11-linked ubiquitin chains to target selected substrates for proteasomal degradation [36]. Furthermore, the posttranslational modification of cyclin B1 by a heterogenous pool of K48-, K11- and K63-linked ubiquitin chains triggers its proteasomal degradation in yeast [37].

Given the sheer diversity of ubiquitin signals that exist in the cell, it remains a daunting task to delineate the functional specificity of each ubiquitin signal in vivo. How do the ubiquitin signals select the correct binding partner at the right place and time to cascade precise signaling within the cellular environment? We envisage that the specificity of ubiquitin signaling in the regulation of a particular molecular or cellular process may be achieved by subcellular compartmentalization of factors important for ubiquitination and differential kinetics of the UBD-ubiquitin recognition (to monoubiquitin and polyubiquitin chains with different linkage and length). For instance, key protein regulators of a particular process may possess UBDs that do not show absolute selectivity toward monoubiquitin or polyubiquitin chains to allow some degree of plasticity in ubiquitin signaling. The fate of these proteins may be critically dependent on the localization and assembly of UBD-containing factors and the availability of enzymes catalyzing the ubiquitination reactions.

\section{Ubiquitin-Proteasome Dysfunction in Disease Pathophysiology and Therapeutic Targeting}

Dysregulation or inactivation of ubiquitin-modifying enzymes may lead to human diseases. For instance, the teratogenic nature of thalidomide, a sedative widely prescribed to pregnant women half a century ago, was recently attributed to its targeting of a component of the CRL that is critical for limb outgrowth and the expression of a fibroblast growth factor (FGF8) during embryonic development [38]. Furthermore, alterations to the physiologic function of ubiquitin-modifying enzymes may enhance or attenuate the responsiveness of patients to therapeutic agents. We herein summarize some of the recent findings in this area.

3.1. Restoration of p53 Function in Cancer. The functional inactivation of $\mathrm{p} 53$, through mutations or abrogation of signaling pathways that regulate its activity, is an almost universal feature of all cancers. Perhaps not surprisingly, much effort has been focused on the restoration of p53 tumor suppressor activity in tumors. This is clearly reflected in the number of ongoing clinical trials and different approaches undertaken to achieve this goal [39-42]. The approaches to reinstate p53 function are broadly categorized into three major groups. While the first group focuses on reestablishing the transcriptional activity of wild-type p53, the second group targets the viability of cancer cells that are highly dependent on their p53 status. Lastly, the third group aims to increase the abundance of wild-type p53 proteins. For instance, a number 
of small molecules, such as JNJ-26854165, MI-219 (also known as AT-219) and the nutlins, bind MDM2 to block its binding to p53. Likewise, RITA (RBPJ interacting and tubulin associated) binds p53 to prevent the latter from binding to MDM2. These small molecules and regulatory proteins yield a similar outcome, one in which p53 is protected from MDM2 ubiquitination and degradation [40,41, 43, 44]. In addition, the development of antisense MDM2 oligonucleotides and small molecules that inhibit USP7 DUB activity have both shown promising results in enhancing MDM2 degradation and stabilizing p53 [39-42, 45, 46].

3.2. Targeting Wnt Signaling in Cancer. The Wnt signal transduction cascade controls a wide variety of biological processes throughout development and adult life of all animals. Since the first reported observation that overexpression of Int-1 (or Wnt1) leads to mammary tumors in mice [47], many labs around the world have shown that the activation of Wnt signaling pathways is intimately associated with cancer stem cell maintenance and cancer progression $[48,49]$. Wnt ligands are secreted, cysteine-rich proteins that interact with Frizzled receptors (Fzd) and the Wnt co-receptors lowdensity lipoprotein receptor-related proteins 5/6 (LPR5/6) to induce the canonical Wnt/ $\beta$-catenin signaling pathway. Furthermore, the binding of Wnt to Fzd alone may initiate noncanonical Wnt signaling, such as the planar cell polarity pathway (PCP).

These Wnt signaling pathways are regulated by a plethora of mechanisms, including the classical regulation of $\beta$ catenin turnover by the UPS machinery. In the absence of Wnt ligands, adenomatous polyposis coli (APC) associates with Axin and the kinases GSK3 and CK1 $\alpha$ (also known as the $\beta$-catenin destruction complex) to foster the phosphorylation of free cytosolic $\beta$-catenin at specific serine/threonine residues creating a phosphodegron motif. Recognition of this phosphodegron by the SCF- $\beta$-TrCP ubiquitin ligase complex leads to the modification of $\beta$-catenin with degradative polyubiquitin, culminating in its proteasomal destruction [50-55]. Notably, the Axin-bound APC appears to be modified predominantly with K63-linked ubiquitin chains in unstimulated HEK293 cells. Wnt3a stimulation results in a loss of K63-polyubiquitin adducts from APC in a timedependent manner, resulting in the dissociation of Axin from APC and the stabilization of cytosolic $\beta$-catenin [56]. During Wnt-driven cancer progression, the stability of Axin appears to be regulated by tankyrase-dependent ubiquitination. In a high throughput screen to identify a small molecule that interfered with Wnt-stimulated transcription, XAV939 exhibited specific inhibition of tankyrases 1 and 2 (TNKS1/2) to stabilize Axin. Importantly, XAV939 showed significant growth inhibition of the APC-defective human colorectal carcinoma cell lines [57]. As ubiquitinated Axin is directly processed by the USP34 ubiquitin protease [58], the development of USP34 inhibitors may offer a novel therapeutic opportunity for Wnt-driven cancers.

ZNRF3 was identified in a genetic screen for $\beta$-catenin gene targets that are potentially involved in the suppression of Wnt signaling. ZNRF3 encodes an E3 ubiquitin ligase with a transmembrane domain and a cytoplasmic RING finger domain. Overexpression of ZNRF3 suppressed $\beta$ catenin-dependent transcription, while silencing of ZNRF3 or overexpression of a dominant negative form of ZNRF3 that lacked the RING finger domain increased LRP6 and Frizzled levels at the membrane and enhanced $\beta$-catenin activation. Importantly, ZNRF3 was found to form a complex with Fzd and LRP6 for ubiquitinating and earmarking Fzd for degradation. Thus, ZNRF3-mediated inhibition of Wnt signaling involves the degradation of Wnt receptors [59]. Given the central role of Wnt signaling in embryonic development and cancer, ZNRF3 is an attractive cancer therapeutic target worth pursuing further.

More recently, the c-Cbl ubiquitin E3 ligase has been shown to target nuclear active $\beta$-catenin to achieve suppression of Wnt signaling in endothelial cells [60]. Thus, enhancement of c-Cbl E3 ligase activity by small molecules may provide an alternative route to perturb the growth of $\mathrm{Wnt} / \beta$-catenin-driven cancers of endothelial origin, although one should remain cautious of any unexpected side effects brought about by the accumulation of other c-Cbl-regulated proteins.

\subsection{Targeting Other Oncoproteins in Cancer. Oncoproteins,} such as the inhibitor of apoptosis (IAP) proteins, have been shown to be frequently upregulated in tumors for the maintenance of cancer cell survival. While ML-IAP exhibits a strong cancer-expression bias, the expression of XIAP and c-IAP1 and c-IAP2 is associated with poor prognosis in a number of tumor types $[61,62]$. The c-IAP1 and c-IAP2 genes are amplified in several human and murine tumor types, suggesting that c-IAP proteins may be good targets of cancer therapeutics $[63,64]$. In a significant proportion of extranodal non-Hodgkin mucosa-associated lymphoid tissue lymphoma translocation (MALT) lymphomas, the $\mathrm{t}(11 ; 18)$ (q21;q21) chromosomal translocation that fuses the $\mathrm{N}$-terminal region of c-IAP2 with the central and C-terminal portion of MALT1 (also known as paracaspase) has been described [63, 65]. The c-IAP2-MALT1 fusion protein constitutively activates the NF- $\kappa$ B pathway to enhance prosurvival and proinflammatory signaling, to promote cancer progression, and to induce resistance to anticancer therapies [66]. Among several innovative strategies to target the IAP proteins thus far, the use of lowmolecular-weight IAP antagonists has attracted the most attention [67].

Low-molecular-weight IAP antagonists mimic the Nterminal end of active SMAC protein and bind select BIR domains of IAP proteins with relatively high affinities to promote cell death and inhibit tumor growth in vivo [67]. Notably, IAP antagonists appear to trigger conformational changes in c-IAP proteins to increase their ubiquitin E3 ligase activity [67-69]. This burst of ubiquitin E3 ligase activity induces rapid autoubiquitination and proteasomal degradation of c-IAP proteins [69-71]. The absence of c-IAP proteins, in turn, triggers NF- $\kappa \mathrm{B}$-inducing kinase (NIK) accumulation and the consequent activation of the noncanonical NF- $\kappa \mathrm{B}$ pathway. TNF $\alpha$, a by-product of the activation of these signaling events, induces TNFR1-mediated signaling to kill cancer cells $[69,71,72]$.

The emerging role of the UPS as a key component of the angiogenic switch in cancer and other types of pathologic 
angiogenesis also highlights the importance of investigating this pathway in the context of angiogenesis. Activation of phospholipase $\mathrm{C} \gamma 1$ (PLC $\gamma 1$ ) in endothelial cells is considered to be one of the main mediators of the angiogenic signaling of VEGFR-2 and tumorigenesis. PLC $\gamma 1$ is a multidomain protein that consists of two $\mathrm{SH} 2$ domains and an $\mathrm{SH} 3$ domain between the catalytic domains. The $\mathrm{SH} 2$ domains recognize phosphotyrosine 1173 (p-Y1173) on mouse VEGFR-2 (corresponding to Y1175 on human VEGFR-2) [73], whereas the SH3 domain recognizes proline-rich sequences (PXXP motifs). The presence of both $\mathrm{N}$ - and C-terminal SH2 domains is required for optimal binding of PLC $\gamma 1$ to VEGFR2 [73]. PLC $\gamma 1$ also interacts with c-Cbl via its proline-rich motif [74]. c-Cbl negatively regulates PLC $\gamma 1$ activation in a proteolysis-independent manner. Instead of targeting PLC $\gamma 1$ for proteasomal degradation, c-Cbl ubiquitinates PLC $\gamma 1$ and suppresses its phosphorylation on Y783 to inactivate PLC $\gamma 1$ [75].

The genetic evidence linking PLC $\gamma 1$ to endothelial cell function and angiogenesis was shown by ablation of PLC $\gamma 1$, which resulted in early embryonic lethality between embryonic days 9.5 and 10.5 due to significantly impaired vasculogenesis and erythrogenesis [76]. Importantly, pharmacological inhibition of PLC $\gamma 1$ by a small molecule, U73122, inhibits endothelial cell tube formation in vitro [73] and angiogenesis in vivo via a chorioallantoic membrane assay [77]. We speculate that the pharmacological enhancement of c-Cbl E3 ligase activity may provide an additional route to inactivate PLC $\gamma 1$ in pathologic angiogenesis.

In addition, the neddylation of the Cullin proteins with NEDD8 regulates the activity of CRLs [78, 79]. Like ubiquitin modifications, NEDD8 conjugation relies on an enzymatic cascade involving initial ATP-dependent activation prior to covalent NEDD8 transfer onto substrates [80, 81]. NEDD8activating enzyme (NAE) consists of NAE1 (ULA1, APPBP1) and UBA3 (UBE1C), with the latter containing the ATP binding pocket and catalytic cysteine $[82,83]$. Given that neddylation of CRLs triggers structural rearrangements associated with enhanced ubiquitin modification of CRL-bound protein substrates [84, 85], the inhibition of NAE has thus emerged as a promising approach to treat cancers via the adenosine sulfamate analog MLN4924. MLN4924 rapidly eliminates CRL neddylation, leading to CRL substrate accumulation and DNA re-replication prior to cancer cell apoptosis [86]. Importantly, MLN4924 regresses tumor growth in mouse xenograft studies and appears to be well tolerated at different doses and treatment regimens. However, the recent work of Toth and coworkers revealed a potential pitfall of this therapeutic strategy [87]. They identified that a singlenucleotide transition of alanine 171 to threonine (A171T) of the NAE subunit UBA3 reduces the enzyme's affinity for MLN4924 and ATP while increasing NEDD8 activation at physiological ATP concentrations. Notably, expression of $\mathrm{UBA}^{\mathrm{A} 171 \mathrm{~T}}$ is sufficient to decrease MLN4924 sensitivity of naive HCT116 cells. These data suggest that the on-target potency of MLN4924 selects for a point mutation in NAE that overcomes the molecule's inhibitory effects, allowing cancer cell survival.
3.4. UPS-Dependent Regulation of Unfolded Proteins in Cancer and Neurodegenerative Diseases. Targeting the UPS as a novel anticancer strategy has been validated by the rapid approval of the US Food and Drug Administration (FDA) of bortezomib (Velcade; Millennium Pharmaceuticals) for the treatment of multiple myeloma and mantle cell lymphoma [88]. Bortezomib and other protease inhibitors, such as carfilzomib or NPI-0052, induce the death of cancer cells through a multitude of cellular pathways. These include stabilization of proapoptotic BCL-2 family members, inhibition of canonical and noncanonical NF- $\kappa \mathrm{B}$ pathways, disruption of tumorstromal interactions, and the accumulation of misfolded proteins [89]. In addition, perturbation of proteasomal activity may overwhelm the quality-control machinery of these malignant cells to induce cell death [90]. While proteasome inhibition is desirable in cancer therapy, the enhancement of proteasome activity appears to be favorable in neurodegenerative diseases. Notably, cells of the neuromuscular origin are particularly sensitive to the aberrant accumulation of oxidized and/or misfolded proteins [88]. Given that the UPS is one of the main routes used to degrade aggregated and misfolded proteins in these neuromuscular cells, enhanced proteasomal activity via inhibition of the proteasome-associated DUB USP14 with the small-molecule inhibitor IU1 may turn out to be a promising therapeutic strategy for a growing list of neurodegenerative diseases induced by proteotoxicity [91].

Apart from targeting USP14, others have also focused on the modulation of the chaperone function of CDC48/p97. The highly conserved CDC48/p97 ATPase plays an important role in the endoplasmic reticulum-associated degradation (ERAD) pathway by hydrolyzing ATP required for the export of ubiquitinated substrates to the cytosol for proteasomedependent degradation. Inhibition of CDC48/p97 has been shown to induce unfolded protein accumulation and, ultimately, cell death. CDC48/p97 missense mutations are found in a genetic form of human dementia. Furthermore, CDC48/p97 was found in ubiquitinated inclusions in affected neurons of amyotrophic lateral sclerosis and Parkinson's disease. Importantly, CDC48/p97 is also overexpressed in multiple cancers, suggesting that this chaperone protein plays diverse and essential cellular roles. Thus, the identification of compounds that selectively target CDC48/p97 function would serve to mitigate human diseases associated with the dysregulation of CDC48p97. To date, DBeQ and ML080 (CID-25110544) have been recently reported to be cell active and reversible inhibitors of CDC48/p97 [92, 93]. Besides impairing both ubiquitin-dependent and autophagic protein clearance pathways in the cell, $\mathrm{DBeQ}$ potently inhibits cancer cell growth, and it exhibits the property of more rapidly mobilizing the executioner caspases- 3 and -7 than a proteasome inhibitor.

3.5. Proteasome Dysfunction in Cardiac Diseases. Proteinopathies are a family of diseases caused by the deficiency in misfolded protein clearance in the cell. The most widely studied cardiac proteinopathy is desmin-related cardiomyopathy (DRC), which is characterized by the inability to deliver ubiquitinated proteins to the $20 \mathrm{~S}$ proteasome as a result 
of reduced protein abundance of critical $20 \mathrm{~S}$ proteasome subunits, like Rpt3 and Rpt5 [94, 95]. Importantly, a growing list of reports suggests that a large subset of common heart diseases likely share pathogenic mechanisms with cardiac proteinopathy. For instance, aberrant protein aggregates have been frequently observed in the pressure-overloaded mouse heart [96]. Furthermore, recent studies on human hearts with end-stage heart failure of common etiologies have unveiled a high prevalence of aberrant protein aggregates that exist as preamyloid oligomers in the diseased hearts [96, 97]. We envisage that strategies to enhance or re-establish UPS function may improve the outcome of many human heart diseases.

\subsection{Proteolysis Targeting Chimeric Molecules (PROTACs). To} target proteins, at will, to the ubiquitin-proteasome pathway, Sakamoto and coworkers pioneered the development of chimeric molecules (referred to as PROTACs) that link a desired target protein to a ubiquitin ligase [98]. PROTACs are structurally comprised of two recognition motifs separated by a linker. One recognition motif is a small-molecule ligand for the target protein of interest, and the other recognizes a specific E3 ligase. Interaction of these motifs with their binding partners results in polyubiquitination of the target protein, which is subsequently subjected to proteasomal degradation. The same E3 ligase recognition motif can be attached to a variety of small-molecule ligands, thereby generalizing this approach to a broad spectrum of proteins.

PROTACs serve as useful tools for the elucidation of protein function and therapeutic applications by inducing proteolytic degradation of their target proteins [99]. For instance, PROTAC-induced degradation of a particular protein followed by determination of the associated phenotypic changes can provide valuable information about the function of the protein of interest. While the first-generation PROTACs were successfully developed using an E3 ubiquitin ligase-recognizing motif derived from $\mathrm{I} \kappa \mathrm{B} \alpha$, they were relatively impermeable to the cell [98]. Its cell permeability property was significantly improved by adopting an HIF- $1 \alpha$ peptide fragment as an E3 ubiquitin ligase recognition motif in the design of the second-generation PROTACs [100-102]. Thus far, androgen receptor (AR) [103] and estrogen receptor (ER) targeting PROTACs [104-106] have been developed, demonstrating their therapeutic potential in the treatment of AR-responsive prostate and ER-positive breast cancers $[103,107,108]$. In addition, PROTACs targeting methionine aminopeptidase-2 (MetAP-2) [98], the aryl hydrocarbon receptor $[109,110]$, and cellular retinoic acid-binding proteins (CRABPs) [111] have also been developed.

\section{Concluding Remarks}

A tight regulation of protein turnover by ubiquitination influences many signaling events and the survival of normal and diseased cells. Our understanding of these processes is far from complete, and a number of important questions remain to be answered. These include the identification of new ubiquitin ligases and DUBs that regulate protein stability, the spatial and temporal nature of ubiquitination in various biological processes, and its relationship with other posttranslational modifications. With a better understanding of the ubiquitin networks and their functions, we can develop novel targeted approaches to treat human diseases that are exquisitely dependent on the dysregulation of these ubiquitin pathways. In general, these should be increasingly specific and personalized and therefore more clinically effective and associated with minimal side effects from off-target effects.

\section{Conflict of Interests}

The authors declare that they have no conflict of interests.

\section{Authors' Contribution}

Jit Kong Cheong wrote the paper, and Stephen I-Hong Hsu edited the paper.

\section{Acknowledgments}

Jit Kong Cheong is supported by the National Medical Research Council (NMRC) New Investigator Grant (NMRC/ BNIG/1078/2012). Stephen I-Hong Hsu is a Departmental Associate at the Sid Martin Biotechnology Institute of the University of Florida.

\section{References}

[1] K. Haglund and I. Dikic, "The role of ubiquitylation in receptor endocytosis and endosomal sorting," Journal of Cell Science, vol. 125, part 2, pp. 265-275, 2012.

[2] R. J. Deshaies and C. A. P. Joazeiro, "RING domain E3 ubiquitin ligases," Annual Review of Biochemistry, vol. 78, pp. 399-434, 2009.

[3] V. Kirkin, D. G. McEwan, I. Novak, and I. Dikic, "A role for ubiquitin in selective autophagy," Molecular Cell, vol. 34, no. 3, pp. 259-269, 2009.

[4] C. Raiborg and H. Stenmark, "The ESCRT machinery in endosomal sorting of ubiquitylated membrane proteins," Nature, vol. 458, no. 7237, pp. 445-452, 2009.

[5] H. D. Ulrich and H. Walden, "Ubiquitin signalling in DNA replication and repair," Nature Reviews Molecular Cell Biology, vol. 11, no. 7, pp. 479-489, 2010.

[6] K. Wickliffe, A. Williamson, L. Jin, and M. Rape, "The multiple layers of ubiquitin-dependent cell cycle control," Chemical Reviews, vol. 109, no. 4, pp. 1537-1548, 2009.

[7] J. M. Winget and T. Mayor, "The diversity of ubiquitin recognition: hot spots and varied specificity," Molecular Cell, vol. 38, no. 5, pp. 627-635, 2010.

[8] C. M. Pickart and D. Fushman, "Polyubiquitin chains: polymeric protein signals," Current Opinion in Chemical Biology, vol. 8, no. 6, pp. 610-616, 2004.

[9] D. Finley, "Recognition and processing of ubiquitin-protein conjugates by the proteasome," Annual Review of Biochemistry, vol. 78, pp. 477-513, 2009.

[10] D. Finley, A. Ciechanover, and A. Varshavsky, "Ubiquitin as a central cellular regulator,” Cell, vol. 116, supplement 2, pp. S29S32, 2004. 
[11] I. Dikic, S. Wakatsuki, and K. J. Walters, "Ubiquitin-binding domains from structures to functions," Nature Reviews Molecular Cell Biology, vol. 10, no. 10, pp. 659-671, 2009.

[12] A. Bremm, S. M. V. Freund, and D. Komander, "Lysl1-linked ubiquitin chains adopt compact conformations and are preferentially hydrolyzed by the deubiquitinase Cezanne," Nature Structural and Molecular Biology, vol. 17, no. 8, pp. 939-947, 2010.

[13] F. Ikeda and I. Dikic, "Atypical ubiquitin chains: new molecular signals. "Protein modifications: beyond the usual suspects" review series," EMBO Reports, vol. 9, no. 6, pp. 536-542, 2008.

[14] K. Iwai and F. Tokunaga, "Linear polyubiquitination: a new regulator of NF- $\kappa$ B activation,” EMBO Reports, vol. 10, no. 7, pp. 706-713, 2009.

[15] M. L. Matsumoto, K. E. Wickliffe, K. C. Dong et al., "K11linked polyubiquitination in cell cycle control revealed by a K11 linkage-specific antibody," Molecular Cell, vol. 39, no. 3, pp. 477484, 2010.

[16] H. Richly, M. Rape, S. Braun, S. Rumpf, C. Hoege, and S. Jentsch, "A series of ubiquitin binding factors connects CDC48/p97 to substrate multiubiquitylation and proteasomal targeting," Cell, vol. 120, no. 1, pp. 73-84, 2005.

[17] K. G. Sim, Z. Zang, C. M. Yang, J. V. Bonventre, and S. I.-H. Hsu, "TRIP-Br links E2F to novel functions in the regulation of cyclin E expression during cell cycle progression and in the maintenance of genomic stability," Cell Cycle, vol. 3, no. 10, pp. 1296-1304, 2004.

[18] K. G. Sim, J. K. Cheong, and S. I.-H. Hsu, "The TRIP-Br family of transcriptional regulators is essential for the execution of cyclin E-mediated cell cycle progression," Cell Cycle, vol. 5, no. 10, pp. 1111-1115, 2006.

[19] Z. Zang, L. Gunaratnam, J. K. Cheong et al., "Identification of PP2A as a novel interactor and regulator of TRIP-Brl," Cellular Signalling, vol. 21, no. 1, pp. 34-42, 2009.

[20] J. K. Cheong, L. Gunaratnam, and S. I.-H. Hsu, "CRM1-mediated nuclear export is required for $26 \mathrm{~S}$ proteasome-dependent degradation of the TRIP-Br2 proto-oncoprotein," The Journal of Biological Chemistry, vol. 283, no. 17, pp. 11661-11676, 2008.

[21] D. Thomas and M. Tyers, "Transcriptional regulation: kamikaze activators," Current Biology, vol. 10, no. 9, pp. R341-R343, 2000.

[22] S. E. Salghetti, A. A. Caudy, J. G. Chenoweth, and W. P. Tansey, "Regulation of transcriptional activation domain function by ubiquitin," Science, vol. 293, no. 5535, pp. 1651-1653, 2001.

[23] P. Kaiser, K. Flick, C. Wittenberg, and S. I. Reed, "Regulation of transcription by ubiquitination without proteolysis: Cdc34/SCF(Met30)-mediated inactivation of the transcription factor Met4," Cell, vol. 102, no. 3, pp. 303-314, 2000.

[24] K. Flick, I. Ouni, J. A. Wohlschlegel et al., "Proteolysis-independent regulation of the transcription factor Met 4 by a single Lys 48-linked ubiquitin chain," Nature Cell Biology, vol. 6, no. 7, pp. 634-641, 2004.

[25] K. Flick, S. Raasi, H. Zhang, J. L. Yen, and P. Kaiser, "A ubiquitininteracting motif protects polyubiquitinated Met4 from degradation by the 26 S proteasome," Nature Cell Biology, vol. 8, no. 5 , pp. 509-515, 2006.

[26] D. Grabs, V. I. Slepnev, Z. Songyang et al., “The SH3 domain of amphiphysin binds the proline-rich domain of dynamin at a single site that defines a new SH3 binding consensus sequence," The Journal of Biological Chemistry, vol. 272, no. 20, pp. 1341913425, 1997.
[27] J. M. Seeling, J. R. Miller, R. Gil, R. T. Moon, R. White, and D. M. Virshup, "Regulation of $\beta$-catenin signaling by the $\mathrm{B} 56$ subunit of protein phosphatase 2A," Science, vol. 283, no. 5410, pp. 20892091, 1999.

[28] S. D. Stamenova, M. E. French, Y. He, S. A. Francis, Z. B. Kramer, and L. Hicke, "Ubiquitin binds to and regulates a subset of SH3 domains," Molecular Cell, vol. 25, no. 2, pp. 273-284, 2007.

[29] S. C. Shih, G. Prag, S. A. Francis, M. A. Sutanto, J. H. Hurley, and L. Hicke, "A ubiquitin-binding motif required for intramolecular monoubiquitylation, the CUE domain," The EMBO Journal, vol. 22, no. 6, pp. 1273-1281, 2003.

[30] S. I. Hsu, C. M. Yang, K. G. Sim, D. M. Hentschel, E. O’leary, and J. V. Bonventre, "TRIP-Br: a novel family of PHD zinc finger- and bromodomain-interacting proteins that regulate the transcriptional activity of E2F-1/DP-1," The EMBO Journal, vol. 20, no. 9, pp. 2273-2285, 2001.

[31] J. K. Cheong, L. Gunaratnam, Z. J. Zang et al., "TRIP-Br2 promotes oncogenesis in nude mice and is frequently overexpressed in multiple human tumors," Journal of Translational Medicine, vol. 7, article 8, 2009.

[32] C. W. Liew, J. Boucher, J. K. Cheong et al., "Ablation of TRIP$\mathrm{Br} 2$, a regulator of fat lipolysis, thermogenesis and oxidative metabolism, prevents diet-induced obesity and insulin resistance," Nature Medicine, vol. 19, no. 2, pp. 217-226, 2013.

[33] J. Z. Zhi, G. S. Khe, K. C. Jit, C. M. Yang, S. Y. Chui, and S. I.$\mathrm{H}$. Hsu, "Exploiting the TRIP-Br family of cell cycle regulatory proteins as chemotherapeutic drug targets in human cancer," Cancer Biology and Therapy, vol. 6, no. 5, pp. 712-718, 2007.

[34] J. J. Sims and R. E. Cohen, "Linkage-specific avidity defines the lysine 63-linked polyubiquitin-binding preference of rap80," Molecular Cell, vol. 33, no. 6, pp. 775-783, 2009.

[35] N. Zhang, Q. Wang, A. Ehlinger et al., "Structure of the s5a:k48linked diubiquitin complex and its interactions with rpn13," Molecular Cell, vol. 35, no. 3, pp. 280-290, 2009.

[36] L. Jin, A. Williamson, S. Banerjee, I. Philipp, and M. Rape, "Mechanism of ubiquitin-chain formation by the human anaphase-promoting complex," Cell, vol. 133, no. 4, pp. 653-665, 2008.

[37] D. S. Kirkpatrick, N. A. Hathaway, J. Hanna et al., "Quantitative analysis of in vitro ubiquitinated cyclin B1 reveals complex chain topology," Nature Cell Biology, vol. 8, no. 7, pp. 700-710, 2006.

[38] T. Ito, H. Ando, T. Suzuki et al., "Identification of a primary target of thalidomide teratogenicity," Science, vol. 327, no. 5971, pp. 1345-1350, 2010.

[39] C. J. Brown, C. F. Cheok, C. S. Verma, and D. P. Lane, "Reactivation of p53: from peptides to small molecules," Trends in Pharmacological Sciences, vol. 32, no. 1, pp. 53-62, 2011.

[40] C. F. Cheok, C. S. Verma, J. Baselga et al., "Translating p53 into the clinic," Nature Reviews Clinical Oncology, vol. 8, no. 1, pp. 25-37, 2011.

[41] A. Di Cintio, E. Di Gennaro, and A. Budillon, "Restoring p53 function in cancer: novel therapeutic approaches for applying the brakes to tumorigenesis," Recent Patents on Anti-Cancer Drug Discovery, vol. 5, no. 1, pp. 1-13, 2010.

[42] A. Mandinova and S. W. Lee, "The p53 pathway as a target in cancer therapeutics: obstacles and promise," Science Translational Medicine, vol. 3, no. 64, Article ID 64rvl, 2011.

[43] M. D. Petroski, "The ubiquitin system, disease, and drug discovery," BMC Biochemistry, vol. 9, supplement 1, article S7, 2008. 
[44] B. T. Vu and L. Vassilev, "Small-molecule inhibitors of the p53-MDM2 interaction," Current Topics in Microbiology and Immunology, vol. 348, pp. 151-172, 2011.

[45] F. Colland, E. Formstecher, X. Jacq et al., "Small-molecule inhibitor of USP7/HAUSP ubiquitin protease stabilizes and activates p53 in cells," Molecular Cancer Therapeutics, vol. 8, no. 8, pp. 2286-2295, 2009.

[46] B. Nicholson, J. G. Marblestone, T. R. Butt, and M. R. Mattern, "Deubiquitinating enzymes as novel anticancer targets," Future Oncology, vol. 3, no. 2, pp. 191-199, 2007.

[47] R. Nusse and H. E. Varmus, "Many tumors induced by the mouse mammary tumor virus contain a provirus integrated in the same region of the host genome," Cell, vol. 31, no. 1, pp. 99109, 1982.

[48] H. Clevers and R. Nusse, "Wnt/beta-catenin signaling and disease," Cell, vol. 149, no. 6, pp. 1192-1205, 2012.

[49] R. Nusse and H. Varmus, "Three decades of Wnts: a personal perspective on how a scientific field developed," The EMBO Journal, vol. 31, no. 12, pp. 2670-2684, 2012.

[50] Z. Gao, J. M. Seeling, V. Hill, A. Yochum, and D. M. Virshup, "Casein kinase I phosphorylates and destabilizes the $\beta$-catenin degradation complex," Proceedings of the National Academy of Sciences of the United States of America, vol. 99, no. 3, pp. 1182$1187,2002$.

[51] V. Korinek, N. Barker, P. J. Morin et al., "Constitutive transcriptional activation by a $\beta$-catenin-Tcf complex in APC(-/-) colon carcinoma," Science, vol. 275, no. 5307, pp. 1784-1787, 1997.

[52] P. J. Morin, A. B. Sparks, V. Korinek et al., "Activation of $\beta$ catenin-Tcf signaling in colon cancer by mutations in $\beta$-catenin or APC," Science, vol. 275, no. 5307, pp. 1787-1790, 1997.

[53] B. Rubinfeld, I. Albert, E. Porfiri, C. Fiol, S. Munemitsu, and P. Polakis, "Binding of GSK $3 \beta$ to the APC- $\beta$-catenin complex and regulation of complex assembly," Science, vol. 272, no. 5264, pp. 1023-1026, 1996.

[54] B. Rubinfeld, B. Souza, I. Albert et al., "Association of the APC gene product with $\beta$-catenin," Science, vol. 262, no. 5140, pp. 1731-1734, 1993.

[55] A. R. Hernandez, A. M. Klein, and M. W. Kirschner, "Kinetic responses of beta-catenin specify the sites of Wnt control," Science, vol. 338, no. 6112, pp. 1337-1340, 2012.

[56] H. Tran and P. Polakis, "Reversible modification of adenomatous polyposis coli (APC) with K63-linked polyubiquitin regulates the assembly and activity of the beta-catenin destruction complex," The Journal of Biological Chemistry, vol. 287, no. 34, pp. 28552-28563, 2012.

[57] S. A. Huang, Y. M. Mishina, S. Liu et al., "Tankyrase inhibition stabilizes axin and antagonizes Wnt signalling," Nature, vol. 461, no. 7264, pp. 614-620, 2009.

[58] T. T. H. Lui, C. Lacroix, S. M. Ahmed et al., "The ubiquitinspecific protease USP34 regulates axin stability and $\mathrm{Wnt} / \beta$ catenin signaling," Molecular and Cellular Biology, vol. 31, no. 10, pp. 2053-2065, 2011.

[59] H. X. Hao, Y. Xie, Y. Zhang et al., "ZNRF3 promotes Wnt receptor turnover in an R-spondin-sensitive manner," Nature, vol. 485, no. 7397, pp. 195-200, 2012.

[60] V. Chitalia, S. Shivanna, J. Martorell et al., "c-Cbl, a ubiquitin E3 ligase that targets active beta-catenin-a novel layer of Wnt regulation," The Journal of Biological Chemistry, 2013.

[61] J. N. Dynek, S. M. Chan, J. Liu, J. Zha, W. J. Fairbrother, and D. Vucic, "Microphthalmia-associated transcription factor is a critical transcriptional regulator of melanoma inhibitor of apoptosis in melanomas," Cancer Research, vol. 68, no. 9, pp. 3124-3132, 2008

[62] A. M. Hunter, E. C. LaCasse, and R. G. Korneluk, "The inhibitors of apoptosis (IAPs) as cancer targets," Apoptosis, vol. 12, no. 9, pp. 1543-1568, 2007.

[63] J. Dierlamm, M. Baens, I. Wlodarska et al., “The apoptosis inhibitor gene API2 and a novel 18q gene, MLT, are recurrently rearranged in the $t(11 ; 18)(\mathrm{q} 21 ; \mathrm{q} 21)$ associated with mucosaassociated lymphoid tissue lymphomas," Blood, vol. 93, no. 11, pp. 3601-3609, 1999.

[64] I. Imoto, H. Tsuda, A. Hirasawa et al., "Expression of cIAP1, a target for 11q22 amplification, correlates with resistance of cervical cancers to radiotherapy," Cancer Research, vol. 62, no. 17, pp. 4860-4866, 2002.

[65] P. G. Isaacson, "Update on MALT lymphomas," Best Practice and Research: Clinical Haematology, vol. 18, no. 1, pp. 57-68, 2005.

[66] H. Zhou, M. Du, and V. M. Dixit, "Constitutive NF- $\kappa$ B activation by the $\mathrm{t}(11 ; 18)(\mathrm{q} 21 ; \mathrm{q} 21)$ product in MALT lymphoma is linked to deregulated ubiquitin ligase activity," Cancer Cell, vol. 7, no. 5, pp. 425-431, 2005.

[67] H. Sun, Z. Nikolovska-Coleska, J. Lu et al., "Design, synthesis, and characterization of a potent, nonpeptide, cell-permeable, bivalent smac mimetic that concurrently targets both the BIR2 and BIR3 domains in XIAP," Journal of the American Chemical Society, vol. 129, no. 49, pp. 15279-15294, 2007.

[68] C. Ndubaku, F. Cohen, E. Varfolomeev, and D. Vucic, “Targeting inhibitor of apoptosis proteins for therapeutic intervention," Future Medicinal Chemistry, vol. 1, no. 8, pp. 1509-1525, 2009.

[69] E. Varfolomeev, J. W. Blankenship, S. M. Wayson et al., "IAP antagonists induce autoubiquitination of c-IAPs, NF-kappaB activation, and TNFalpha-dependent apoptosis," Cell, vol. 131, no. 4, pp. 669-681, 2007.

[70] M. J. M. Bertrand, S. Milutinovic, K. M. Dickson et al., "cIAP1 and cIAP2 facilitate cancer cell survival by functioning as E3 ligases that promote RIP1 ubiquitination," Molecular Cell, vol. 30 , no. 6, pp. 689-700, 2008.

[71] J. E. Vince, W. W. Wong, N. Khan et al., "IAP antagonists target cIAP1 to induce TNFalpha-dependent apoptosis," Cell, vol. 131, no. 4, pp. 682-693, 2007.

[72] S. L. Petersen, L. Wang, A. Yalcin-Chin et al., "Autocrine TNFalpha signaling renders human cancer cells susceptible to Smac-mimetic-induced apoptosis," Cancer Cell, vol. 12, no. 5, pp. 445-456, 2007.

[73] R. D. Meyer, C. Latz, and N. Rahimi, "Recruitment and activation of phospholipase $\mathrm{C} \gamma 1$ by vascular endothelial growth factor receptor- 2 are required for tubulogenesis and differentiation of endothelial cells," The Journal of Biological Chemistry, vol. 278, no. 18, pp. 16347-16355, 2003.

[74] A. J. Singh, R. D. Meyer, H. Band, and N. Rahimi, “The carboxyl terminus of VEGFR-2 is required for PKC-mediated downregulation," Molecular Biology of the Cell, vol. 16, no. 4, pp. 21062118, 2005.

[75] A. J. Singh, R. D. Meyer, G. Navruzbekov et al., "A critical role for the E3-ligase activity of c-Cbl in VEGFR-2-mediated PLCgammal activation and angiogenesis," Proceedings of the National Academy of Sciences of the United States of America, vol. 104, no. 13, pp. 5413-5418, 2007. 
[76] H. Liao, T. Kume, C. McKay, M. Xu, J. N. Ihle, and G. Carpenter, "Absence of erythrogenesis and vasculogenesis in Plcg1deficient mice," The Journal of Biological Chemistry, vol. 277, no. 11, pp. 9335-9341, 2002.

[77] R. D. Meyer, D. Husain, and N. Rahimi, "c-Cbl inhibits angiogenesis and tumor growth by suppressing activation of PLC $\gamma 1$," Oncogene, vol. 30, no. 19, pp. 2198-2206, 2011.

[78] D. R. Bosu and E. T. Kipreos, "Cullin-RING ubiquitin ligases: global regulation and activation cycles," Cell Division, vol. 3, article 7, 2008.

[79] M. D. Petroski and R. J. Deshaies, "Function and regulation of cullin-RING ubiquitin ligases," Nature Reviews Molecular Cell Biology, vol. 6, no. 1, pp. 9-20, 2005.

[80] R. N. Bohnsack and A. L. Haas, "Conservation in the mechanism of Nedd8 activation by the human AppBp1-Uba3 heterodimer," The Journal of Biological Chemistry, vol. 278, no. 29, pp. 26823-26830, 2003.

[81] B. A. Schulman and J. Wade Harper, "Ubiquitin-like protein activation by E1 enzymes: the apex for downstream signalling pathways," Nature Reviews Molecular Cell Biology, vol. 10, no. 5, pp. 319-331, 2009.

[82] L. Gong and E. T. H. Yeh, "Identification of the activating and conjugating enzymes of the NEDD8 conjugation pathway," The Journal of Biological Chemistry, vol. 274, no. 17, pp. 12036-12042, 1999.

[83] F. Osaka, H. Kawasaki, N. Aida et al., "A new NEDD8-ligating system for cullin-4A," Genes and Development, vol. 12, no. 15, pp. 2263-2268, 1998.

[84] D. M. Duda, L. A. Borg, D. C. Scott, H. W. Hunt, M. Hammel, and B. A. Schulman, "Structural insights into NEDD8 activation of cullin-RING ligases: conformational control of conjugation," Cell, vol. 134, no. 6, pp. 995-1006, 2008.

[85] F. Osaka, M. Saeki, S. Katayama et al., "Covalent modifier NEDD8 is essential for SCF ubiquitin-ligase in fission yeast," The EMBO Journal, vol. 19, no. 13, pp. 3475-3484, 2000.

[86] T. A. Soucy, P. G. Smith, M. A. Milhollen et al., "An inhibitor of NEDD8-activating enzyme as a new approach to treat cancer," Nature, vol. 458, no. 7239, pp. 732-736, 2009.

[87] J. I. Toth, L. Yang, R. Dahl et al., "A gatekeeper residue for NEDD8-activating enzyme inhibition by MLN4924," Cell Reports, vol. 1, no. 4, pp. 309-316, 2012.

[88] S. Grimm, A. Höhn, and T. Grune, "Oxidative protein damage and the proteasome," Amino Acids, vol. 42, no. 1, pp. 23-38, 2012.

[89] D. J. McConkey and K. Zhu, "Mechanisms of proteasome inhibitor action and resistance in cancer," Drug Resistance Updates, vol. 11, no. 4-5, pp. 164-179, 2008.

[90] A. G. Eldridge and T. O’Brien, “Therapeutic strategies within the ubiquitin proteasome system," Cell Death and Differentiation, vol. 17, no. 1, pp. 4-13, 2010.

[91] B. Lee, M. J. Lee, S. Park et al., "Enhancement of proteasome activity by a small-molecule inhibitor of USP14," Nature, vol. 467, no. 7312, pp. 179-184, 2010.

[92] S. J. Brown, T. F. Chou, R. Deshaies et al., "Probe report for P97/cdc48 inhibitors," in Probe Reports from the NIH Molecular Libraries Program, National Center for Biotechnology Information, Bethesda, Md, USA, 2010.

[93] T. Chou, S. J. Brown, D. Minond et al., "Reversible inhibitor of p97, DBeQ, impairs both ubiquitin-dependent and autophagic protein clearance pathways," Proceedings of the National
Academy of Sciences of the United States of America, vol. 108, no. 12, pp. 4834-4839, 2011.

[94] Q. Chen, J. Liu, K. M. Horak et al., "Intrasarcoplasmic amyloidosis impairs proteolytic function of proteasomes in cardiomyocytes by compromising substrate uptake," Circulation Research, vol. 97, no. 10, pp. 1018-1026, 2005.

[95] J. Liu, M. Tang, R. Mestril, and X. Wang, "Aberrant protein aggregation is essential for a mutant desmin to impair the proteolytic function of the ubiquitin-proteasome system in cardiomyocytes," Journal of Molecular and Cellular Cardiology, vol. 40, no. 4, pp. 451-454, 2006.

[96] P. Tannous, H. Zhu, A. Nemchenko et al., "Intracellular protein aggregation is a proximal trigger of cardiomyocyte autophagy," Circulation, vol. 117, no. 24, pp. 3070-3078, 2008.

[97] A. Sanbe, H. Osinska, C. Villa et al., "Reversal of amyloidinduced heart disease in desmin-related cardiomyopathy," Proceedings of the National Academy of Sciences of the United States of America, vol. 102, no. 38, pp. 13592-13597, 2005.

[98] K. M. Sakamoto, K. B. Kim, A. Kumagai, F. Mercurio, C. M. Crews, and R. J. Deshaies, "Protacs: chimeric molecules that target proteins to the Skp1-Cullin-F box complex for ubiquitination and degradation," Proceedings of the National Academy of Sciences of the United States of America, vol. 98, no. 15, pp. 8554-8559, 2001.

[99] K. M. Sakamoto, "Protacs for treatment of cancer," Pediatric Research, vol. 67, no. 5, pp. 505-508, 2010.

[100] J. S. Schneekloth Jr., F. N. Fonseca, M. Koldobskiy et al., "Chemical genetic control of protein levels: selective in vivo targeted degradation," Journal of the American Chemical Society, vol. 126, no. 12 , pp. 3748-3754, 2004.

[101] D. Zhang, S. H. Baek, A. Ho, and K. Kim, "Degradation of target protein in living cells by small-molecule proteolysis inducer," Bioorganic \& Medicinal Chemistry Letters, vol. 14, no. 3, pp. 645648, 2004.

[102] D. Zhang, S.-H. Baek, A. Ho, H. Lee, Y. S. Jeong, and K. Kim, "Targeted degradation of proteins by small molecules: a novel tool for functional proteomics," Combinatorial Chemistry and High Throughput Screening, vol. 7, no. 7, pp. 689-697, 2004.

[103] A. Rodriguez-Gonzalez, K. Cyrus, M. Salcius et al., "Targeting steroid hormone receptors for ubiquitination and degradation in breast and prostate cancer," Oncogene, vol. 27, no. 57, pp. 72017211, 2008.

[104] K. Cyrus, M. Wehenkel, E. Choi et al., "Impact of linker length on the activity of PROTACs," Molecular BioSystems, vol. 7, no. 2, pp. 359-364, 2011.

[105] K. Cyrus, M. Wehenkel, E. Choi, H. Lee, H. Swanson, and K. Kim, "Jostling for position: optimizing linker location in the design of estrogen receptor-targeting PROTACs," ChemMedChem, vol. 5, no. 7, pp. 979-985, 2010.

[106] K. Cyrus, M. Wehenkel, E. Choi, H. Swanson, and K. Kim, "Two-headed PROTAC: an effective new tool for targeted protein degradation," ChemBioChem, vol. 11, no. 11, pp. 15311534, 2010.

[107] K. M. Sakamoto, "Chimeric molecules to target proteins for ubiquitination and degradation," Methods in Enzymology, vol. 399, article no. 54, pp. 833-847, 2005.

[108] K. M. Sakamoto, K. B. Kim, R. Verma et al., "Development of Protacs to target cancer-promoting proteins for ubiquitination and degradation," Molecular \& Cellular Proteomics, vol. 2, no. 12, pp. 1350-1358, 2003. 
[109] H. Lee, D. Puppala, E. Choi, H. Swanson, and K. Kim, “Targeted degradation of the aryl hydrocarbon receptor by the PROTAC approach: a useful chemical genetic tool," ChemBioChem, vol. 8, no. 17, pp. 2058-2062, 2007.

[110] D. Puppala, H. Lee, B. K. Kyung, and H. I. Swanson, "Development of an aryl hydrocarbon receptor antagonist using the proteolysis-targeting chimeric molecules approach: a potential tool for chemoprevention," Molecular Pharmacology, vol. 73, no. 4, pp. 1064-1071, 2008.

[111] Y. Itoh, M. Ishikawa, M. Naito, and Y. Hashimoto, "Protein knockdown using methyl bestatin-ligand hybrid molecules: design and synthesis of inducers of ubiquitination-mediated degradation of cellular retinoic acid-binding proteins," Journal of the American Chemical Society, vol. 132, no. 16, pp. 5820-5826, 2010. 


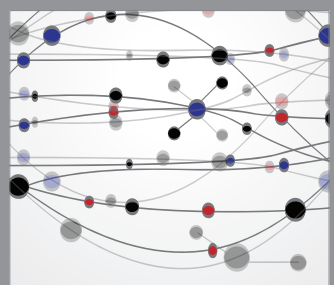

The Scientific World Journal
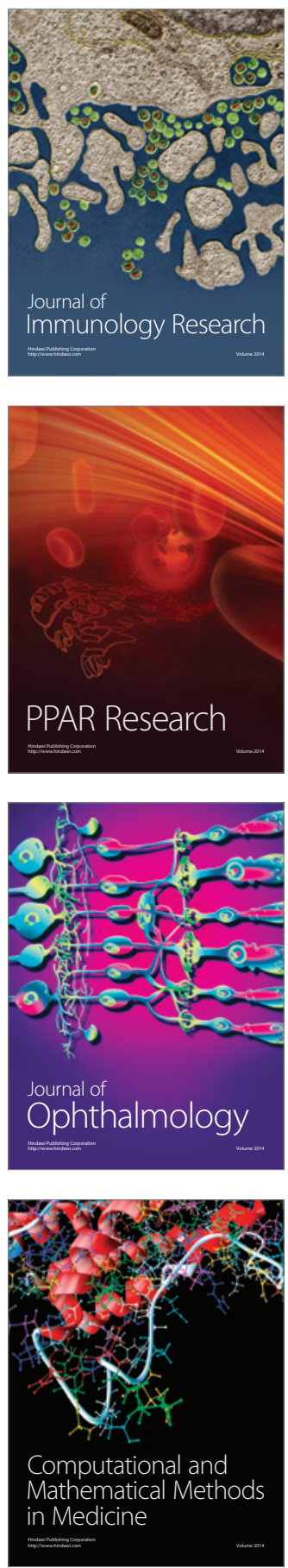

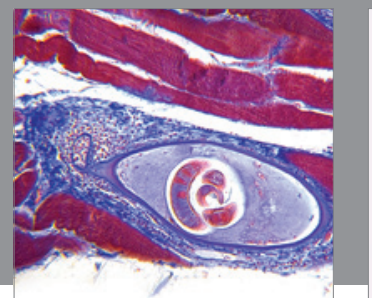

Gastroenterology

Research and Practice
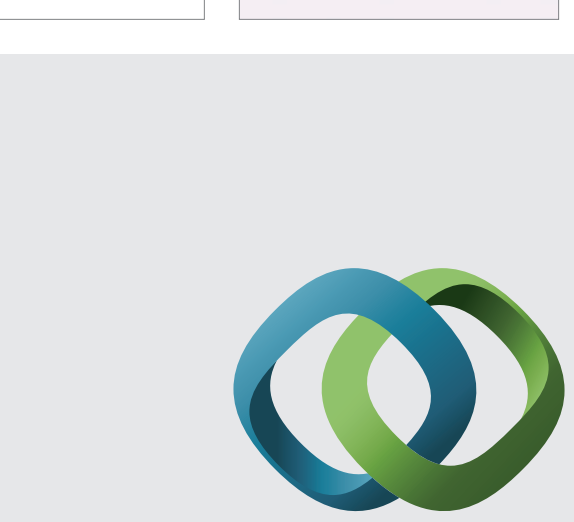

\section{Hindawi}

Submit your manuscripts at

http://www.hindawi.com
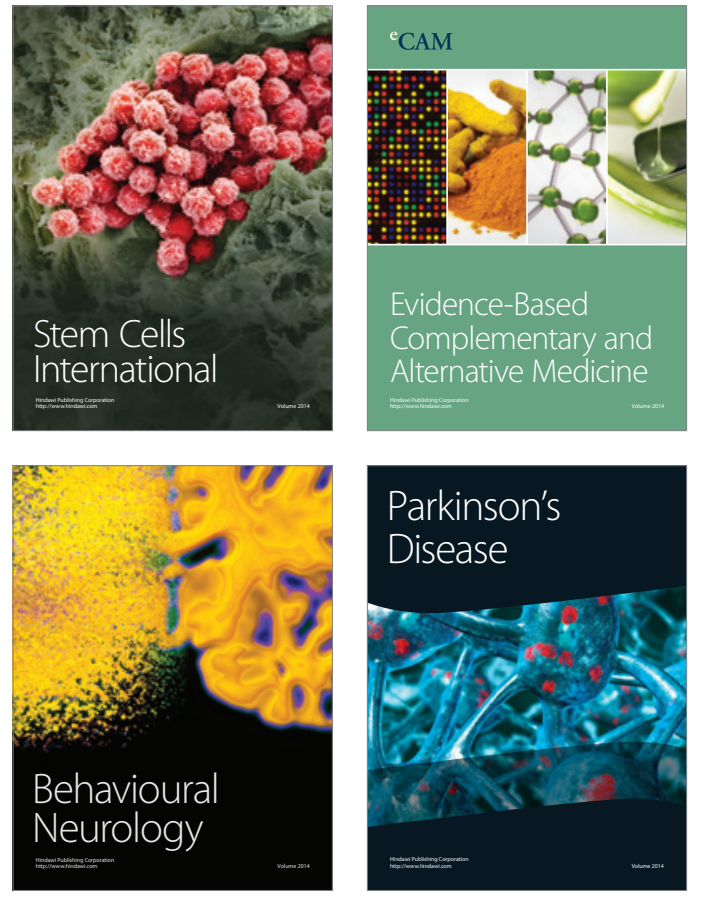
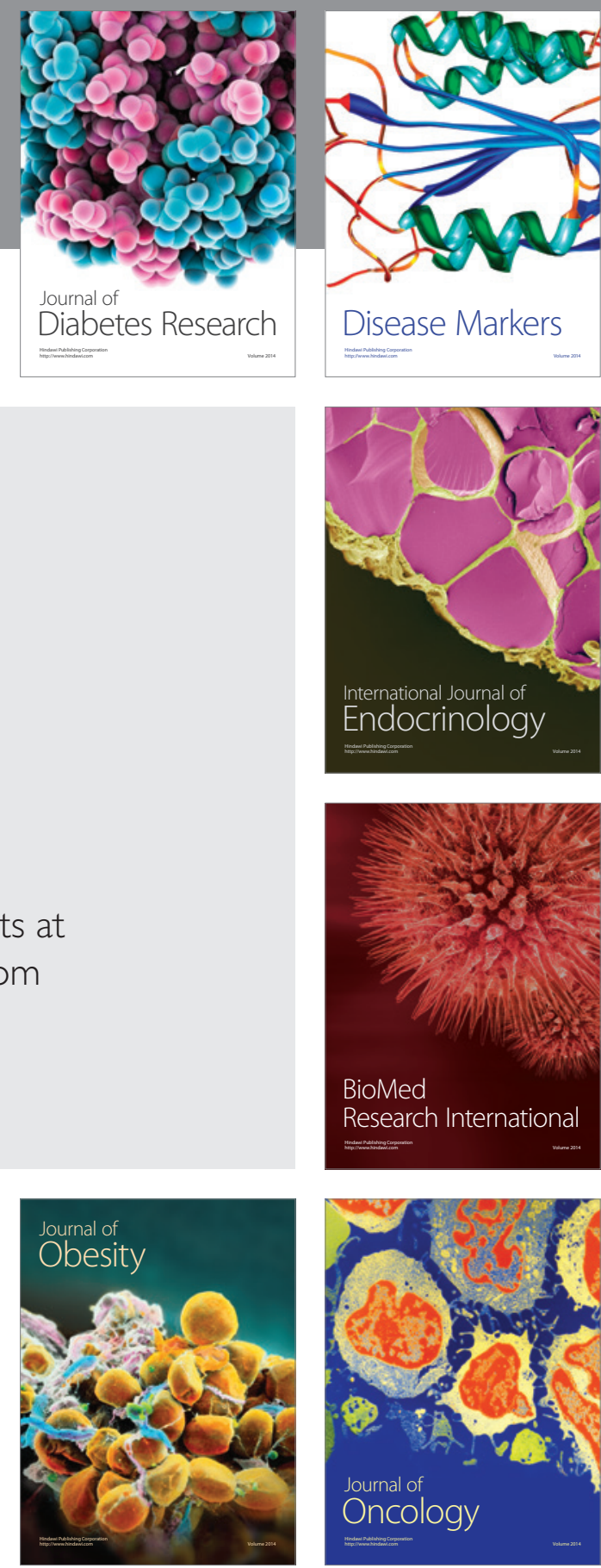

Disease Markers
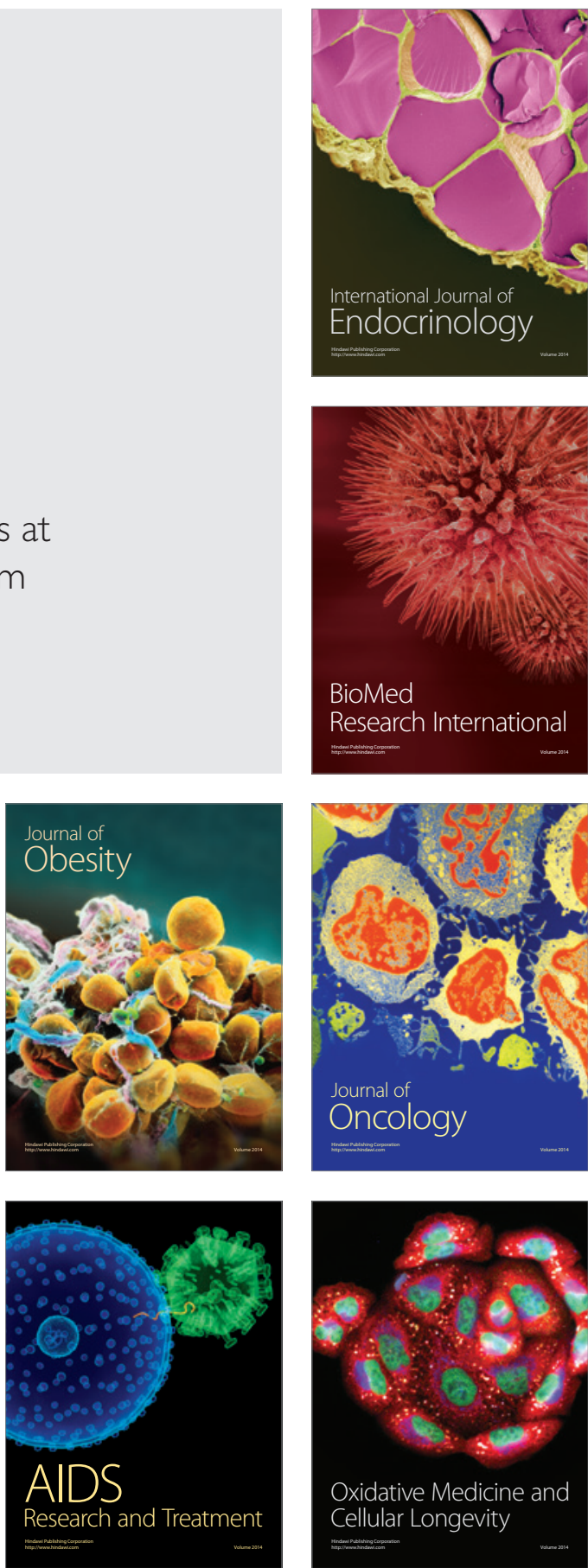